\title{
Turismo y la lucha contra la pobreza en el Perú. ANÁLISIS DE DOS CASOS REGIONALES
}

\author{
Jessica Figueroa Pinedo \\ jessicafigueroapinedo@gmail.com \\ Laboratori Multidisciplinar de Recerca en Turisme \\ Universitat de Girona, España
}

La importancia que está adquiriendo la actividad turística en el Perú invita a analizar su estrecha relación con la pobreza, pues las prácticas turísticas se desenvuelven en regiones con persistentes niveles de pobreza y desigualdad. El objetivo del presente artículo es contribuir al debate sobre las posibilidades del sector turismo de reducir la pobreza en regiones pobres. Para ello, se presentan dos casos de estudios en el Perú: Cuzco, en el sur andino y Trujillo (La Libertad) en la costa norte. A partir de datos secundarios de las fuentes oficiales se procede a un análisis en profundidad de la actividad turística y su impacto socioeconómico en cada región. Los resultados llaman la atención hacia los problemas sociales y las dificultades que tienen estas regiones en lograr que los beneficios de la actividad turística repercutan en la población local.

Palabras clave: Turismo, pobreza, desarrollo regional, Perú.

\section{Tourism and fighting poverty in Peru. TWO REGIONAL CASE STUDIES}

The current importance achieved by the tourism sector in Peru begs for an analysis of its close relationship with poverty, since tourist practices are developed in regions with persistent levels of poverty and inequality. The objective of this article is to contribute to the debate on the possibilities of the tourism sector to reduce poverty in poor regions. For this purpose, it analyses two case studies in Peru: Cuzco, in the Southern Andes and Trujillo (La Libertad), in the North coast. An in-depth analysis of tourism activity and its socioeconomic impact in each region is carried out based on secondary data from official sources, Findings draw attention to the difficulties that these regions have in making the benefits of tourist activity have a positive impact on the local population.

Keywords: tourism development, poverty, regional development, Peru.

Fecha de recepción: 12 de marzo de 2019. Fecha de aceptación: 20 de diciembre de 2019

CÓMO CITAR: Figueroa-Pinedo, J. (2020). Turismo y la lucha contra la pobreza en el Perú. Análisis de dos casos regionales. Dimensiones Turísticas, 4(6),39-62. https://doi.org/10.47557/SCU01957 


\section{Introducción}

a reducción de la pobreza se ha convertido en uno de los principales retos del siglo XXI a nivel internacional y actualmente constituye uno de los principales Objetivos de Desarrollo Sostenible (ODS) propuestos por la Organización de las Naciones Unidas (ONU) para el 2030. En ese sentido, la importancia mundial del turismo como actividad económica ha llevado a los organismos internacionales liderados por la Organización Mundial de Turismo (OMT) a promocionarlo como una herramienta indispensable para la atenuación de la pobreza en países en vías de desarrollo (OMT, 2003). Al asumir ese reto, la OMT trató de enfocar el turismo como un instrumento de reducción de la pobreza por su capacidad de generar empleo y definió correctamente el problema. Se trata de centrar las prácticas turísticas en términos de desarrollo sostenible de las poblaciones servidas por el flujo de turistas y de sacar el máximo provecho de la inversión económica y financiera que ellas suponen, poniendo en valor el territorio, la diversidad cultural y promover una mayor vinculación de los pobres con el mercado turístico local (OMT, 2005; 2010; UNDP, 2011).

Otros organismos multilaterales, como el Banco Mundial, el Banco Interamericano de Desarrollo (BID), el Fondo Monetario Internacional (FMI), el Programa de las Naciones Unidas para el Desarrollo (PNUD, UNDP por sus siglas en inglés) han demostrado un mayor interés en usar el turismo como herramienta de desarrollo y lucha contra la pobreza en sus proyectos, programas, subvenciones y préstamos a países en desarrollo. Por ejemplo, el Banco Mundial ha tenido un importante rol en el desarrollo del turismo y la preservación de monumentos arqueológicos desde la década de 1960 (Hawkins y Mann, 2007). Las organizaciones bilaterales también han demostrado un renovado interés en el turismo, por ejemplo, las agencias internacionales de desarrollo, tales como: I'Agence Canadienne de Développement International $(A C D /)$, la Agencia Española de Cooperación Internacional para el Desarrollo (AECID) o SNV Netherlands Development Organization, todas involucradas con la actividad turística desde diferentes perspectivas pero con el objetivo de solucionar el problema de la pobreza y mejorar las condiciones de vida de muchas poblaciones de países en desarrollo (Lima et al. 2012; Hummel y Van der Duim, 2012). Sin embargo, pese a los esfuerzos de ayudas, programas y cooperación internacional, los avances en el cumplimiento de los objetivos de reducción de la pobreza a nivel mundial aún son limitados y generan muchos debates sobre su efectividad real (Lima, 2014; Gascón et al., 2013; Scheyvens, 2007). 
En ese contexto, el Estado peruano, adoptando las directrices de la OMT, ha orientado el desarrollo de la actividad turística hacia la lucha contra la pobreza. Un reflejo de ello, es el Plan Estratégico Nacional de Turismo (Pentur), uno de los planes más importantes realizados en el Perú sobre el desarrollo regional y ordenamiento turístico, bajo la supervisión del Ministerio de Comercio Exterior y Turismo (Mincetur), y que tiene como objetivo principal dirigir y promover el desarrollo competitivo de la actividad turística del Perú, con políticas orientadas hacia el desarrollo sostenible, la lucha contra la pobreza y la inclusión social (Sariego y García, 2008; Mincetur, 2016).

En efecto, el crecimiento del turismo en Perú ha sido muy positivo en la última década; según datos del referido Ministerio, la llegada de turistas internacionales aumentó de 1.4 millones en el 2004 a 4.4 millones en el 2018. Por consiguiente, la actividad turística también ha adquirido mayor importancia para la economía nacional. Acorde con Mincetur, los ingresos de divisas por turismo receptivo aumentaron de 1.2 millones de dólares en 2004 a 4.9 millones de dólares en 2018, con un aporte de 3.9\% al Producto Interno Bruto (PBI) nacional.

La importancia que adquiere la actividad turística en el Perú invita a analizar su estrecha relación con la pobreza pues las prácticas turísticas se desenvuelven en regiones con persistentes niveles de pobreza y desigualdad. Por una lado, El turismo internacional se concentra en el tradicional circuito sur andino, una región que concentra una gran riqueza histórica y cultural, pero a la vez muchas de sus comunidades experimentan profundas privaciones y desigualdades, principalmente en las zonas altas y montañosas de los Andes (Figueroa et al., 2015). Por otro lado, en la última década el Gobierno peruano busca diversificar la oferta turística poniendo en valor los atractivos turísticos de otras regiones del país, como es el caso de la Costa Norte del Perú, con el fin de presentar un país diverso y renovado para atraer la mirada turística internacional hacía otros destinos (Sariego y García, 2008; González, 2017).

Por consiguiente, el objetivo de este artículo es contribuir al debate sobre las posibilidades que ofrece el sector turístico en reducir la pobreza y lograr que las poblaciones locales se beneficien de las oportunidades del desarrollo turístico de manera equitativa y sostenible. Para ello presentaremos dos casos de estudio en Perú: el caso de Cuzco en el sur andino y Trujillo (Departamento de La Libertad), en la costa norte. Si bien son regiones con sus propias características geográficas, socioeconómicas y niveles de desarrollo turístico diferentes, representan espacios interesantes para este tipo de análisis, con experiencias turísticas excepcionales en términos culturales y 
patrimoniales y, a la vez, poblaciones que sufren las consecuencias de los niveles de pobreza y vulnerabilidad.

Cuzco-Machu Picchu es un destino turístico internacional consolidado hace décadas, que atrae a un turismo de masas. Mientras que Trujillo, en el Departamento de La Libertad, es un destino turístico en pleno auge que está haciendo grandes esfuerzos de promoción y revalorización de su patrimonio arqueológico (Trivelli y Hernández, 2009) para captar también la mirada del turista extranjero y convertirse en el segundo destino turístico internacional en el Perú.

En primer lugar, se presenta un balance de la relación entre turismo y pobreza. Posteriormente, se muestran los casos de estudio analizados a través de los datos secundarios de las fuentes oficiales. Finalmente, el análisis comparativo y las conclusiones.

\section{La relación entre el turismo y pobreza}

Actualmente la pobreza es considerada como un fenómeno multidimensional y complejo de definir porque intervienen múltiples variables y dimensiones (Alkire y Foster, 2009; Herrera, 2002). Los especialistas coinciden en que la pobreza debe ser analizada no solo a partir de los datos económicos, sino que necesita incluir aspectos cualitativos que expliquen las causas reales y profundas de este fenómeno. Como afirma Sen (2000), el análisis no debe centrarse en los niveles de ingresos y consumo sino en la capacidad de los individuos de tener acceso a ellos. En ese sentido, según Dehoorne (2013, p.1, traducción propia), "Iuchar contra la pobreza debe entenderse como la voluntad de construir una 'libertad positiva', de construir las condiciones de poder elegir, tener suficientes recursos -en un sentido ampliopara poder tomar sus propias decisiones en pleno conocimiento y no dictadas por la necesidad".

Por lo tanto, ¿el turismo puede contribuir a reducir la pobreza? Desde el campo académico, el análisis del turismo como instrumento de desarrollo y lucha contra la pobreza ha sido muy cuestionado y se ha generado todo tipo de análisis y debates. Actualmente existe una amplia literatura y un mayor interés sobre el tema: desde visiones muy críticas de los efectos negativos del turismo en los destinos y que cuestionan el discurso triunfalista del mismo como motor de desarrollo, propagado principalmente para favorecer a los grandes empresarios interesados en el negocio turístico (De Kadt, 1991; Jurdao, 1992; Christin, 2008; Gascón y Cañada, 2005), hasta a análisis más moderados que enfatizan los aspectos positivos del turismo sostenible, la con- 
servación de los espacios naturales y la participación e integración de la comunidades locales (Huaita Alfaro, 2012; Scheyvens y Russell, 2012).

Lo cierto es que tanto el turismo como la pobreza son hechos socioculturales que están estrechamente vinculados a las mismas circunstancias o participan en los mismos contextos territoriales. Muchas de las prácticas turísticas se desarrollan en zonas o regiones donde los problemas de acceso a los bienes y servicios elementales escasean o donde los niveles de educación y salud de la población son muy bajos.

Para Jurdao (1992, p. 30), el turismo en algunos casos es una estrategia de desarrollo peligrosamente ambigua. Menciona que, para construir un modelo de articulación del turismo internacional en los destinos turísticos del Tercer Mundo, es preciso tener en consideración dos grupos de factores: primero, entender cómo se presenta la industria y quién se beneficia del desarrollo turístico. Este punto es de vital importancia a la hora de analizar si realmente el turismo constituye un motor de desarrollo en zonas de pobreza. Segundo, es necesario analizar la organización y la estructura comercial de la propia industria turística, en especial el poder de control de ciertos elementos activos. Es decir, si el funcionamiento del mercado turístico es equitativo y si estimula la participación de la población local o solo se concentra en pocas manos.

En esa línea, Urry (2004) comenta que el turismo en países en desarrollo como México, Kenia y Gambia, plantea cuestiones difíciles que no se derivan de procesos internos de dichas sociedades sino de una serie de situaciones externas: cambios tecnológicos como los viajes aéreos baratos; el crecimiento a nivel mundial del capital como grupos hoteleros, agencias de viajes; la búsqueda de nuevos destinos no tradicionales o lo que Urry llama la difusión de la mirada "romántica", y la idea cada vez más difundida de que el turismo es un gran potencial de desarrollo. En ese sentido, vale la pena preguntarse ¿desarrollo para quién? pues, como señala el autor, muchas de las instalaciones e infraestructuras creadas para el turismo serán poco beneficiosas para la población indígena. Lo mismo pasa en el caso de la riqueza generada por la actividad turística se distribuirá de manera muy desigual (Urry, 2004, p. 119).

Resulta muy ilustrativo el análisis de Gascón (2012) sobre el caso de República Dominicana, país sumamente turístico y a la vez paradigmático, pues a pesar del fuerte crecimiento del Producto Interno Bruto gracias a la actividad turística, su población tiene un bajo nivel de vida. 
Por otro lado, como señala (Goodwin, 2013) existen algunos casos de éxito documentados en los que el turismo ha generado beneficios para las comunidades locales; por ejemplo, en África Occidental, en Gambia los agricultores pobres de frutas y verduras se han beneficiado vendiendo a los hoteles.

Lima et al. (2012) examinan el papel del turismo para el mundo en desarrollo a través de la cooperación internacional analizando en concreto el Programa UNWTO Volunteers en Chiapas México. Los autores plantean que para que el turismo sea un instrumento de desarrollo eficaz, estos programas deben considerar los siguientes aspectos: en primer lugar, la participación comunitaria es vital porque produce decisiones más apropiadas. Otro aspecto aspecto señalado es el empoderamiento (empowerment) de los miembros de las comunidades locales, quienes se vuelvan agentes del cambio y capaces de tomar sus propias decisiones sobre la actividad turística. Sin embargo, también mencionan que se requiere el apoyo de los estados en proporcionar la legislación, información y adiestramiento para el éxito de estas iniciativas. Además, Goodwin (2013) añade que, si las iniciativas turísticas establecen asociaciones colaborativas eficaces con un sector privado de éxito, el turismo puede reducir la pobreza.

\section{Casos de estudio}

\subsection{El caso de Cuzco}

El Departamento de Cuzco se encuentra situado en el sur de Perú, su territorio abarca desde las más altas cumbres andinas hasta la selva. La variedad geográfica corresponde también a una gran diversidad de poblaciones y de géneros de vida. En la montaña viven los lugareños, generalmente muy aislados y consagrados al pastoreo de altura, mientras que en los valles, como el Cuzco, los habitantes son muy dinámicos y activos en lo que se refiere a la agricultura; pero en los últimos 30 años se han incorporado a las actividades turísticas y artesanales. Según datos del Instituto Nacional de Estadística e Informática (INEI, 2018a), el departamento tiene una población total de 1205527 habitantes, una de las más importantes del sur andino.

La ciudad de Cuzco (capital del departamento) concentra una enorme riqueza patrimonial y cultural por su condición de capital del Imperio Inca. Posteriormente, tras la llegada de los españoles en 1534, Cuzco se convirtió en una de las ciudades más importantes del Virreinato, donde se mezclan monumentos y templos incas con la arquitectura colonial. En Cuzco se encuentra el atractivo turístico más importante y famoso a nivel internacional: el Santuario Histórico de Machu Picchu, símbolo de las 
culturas prehispánicas de América del Sur e ícono turístico del Perú. Como menciona Valcárcel (2009, p. 29) "es sencillamente impresionante, inolvidable, una visita a Machu Picchu, moderna Meca para las gentes de todas las naciones". Cuzco y Machu Picchu fueron declarados Patrimonio de la Humanidad por la Unesco en 1983, en reconocimiento a sus valores culturales y naturales excepcionales.

\section{Características de las prácticas turísticas cuzqueñas}

Cuzco ha experimentado una serie de cambios sociales y económicos; precisamente, a partir de 1960 cuando se dio el primer gran impulso para el desarrollo de la actividad turística en el Perú con la inversión en infraestructura, restauración y puesta en valor de monumentos arquitectónicos a través del Plan Copesco ${ }^{1}$. Esos hechos cambiaron completamente el paisaje turístico de esta región.

No cabe duda que Cuzco ejerce un gran poder de atracción tanto para el turismo internacional como para el nacional, a partir del interés que despertó el descubrimiento de sus ruinas a inicios del siglo XX. Además, la declaración de Machu Picchu como una de las maravillas del mundo moderno en 2007, significó mayor promoción para la región. Según datos del Observatorio Turístico del Perú, la llegada total de visitantes a Cuzco presenta un crecimiento sostenido desde 1992 de 242 mil 264 visitantes a 1 408146 en 2015, como se aprecia en el cuadro 1.

Cuadro 1. Evolución histórica del número de turistas en el Departamento de Cuzco 1992-2015

\begin{tabular}{|c|c|c|c|}
\hline Año & Turistas extranjeros & Turistas nacionales & Total \\
\hline 1992 & 45734 & 196530 & 242264 \\
\hline 1993 & 69173 & 228118 & 297291 \\
\hline 1994 & 131536 & 256913 & 388449 \\
\hline 1995 & 173894 & 254795 & 428689 \\
\hline 1996 & 216758 & 247415 & 464173 \\
\hline 1997 & 218752 & 248963 & 467715 \\
\hline 1998 & 269762 & 227204 & 496966 \\
\hline 1999 & 330349 & 232070 & 562419 \\
\hline 2000 & 361211 & 218483 & 579694 \\
\hline 2001 & 410448 & 233084 & 643532 \\
\hline 2002 & 366325 & 249908 & 616233 \\
\hline
\end{tabular}

\footnotetext{
1 Este plan nace de la Comisión Especial Perú Unesco (Copesco). Actualmente es una unidad ejecutora del Mincetur para crear proyectos de infraestructura turística a nivel nacional. Para más detalles consultar: https://www. plancopesconacional.gob.pe/
} 
Continuación del cuadro 1.

\begin{tabular}{|c|c|c|c|}
\hline Año & Turistas extranjeros & Turistas nacionales & Total \\
\hline 2003 & 455249 & 241542 & 696791 \\
\hline 2004 & 491822 & 242504 & 734326 \\
\hline 2005 & 528394 & 243466 & 771860 \\
\hline 2006 & 564967 & 244428 & 809395 \\
\hline 2007 & 601540 & 245390 & 846930 \\
\hline 2008 & 638112 & 246351 & 884463 \\
\hline 2009 & 614374 & 247313 & 861687 \\
\hline 2010 & 517123 & 248275 & 765398 \\
\hline 2011 & 674685 & 325876 & 1000561 \\
\hline 2012 & 775423 & 408765 & 1184188 \\
\hline 2013 & 820979 & 420987 & 1241966 \\
\hline 2014 & 857548 & 421200 & 1278748 \\
\hline 2015 & 967266 & 440880 & 1408146 \\
\hline
\end{tabular}

Fuente: Observatorio Turístico del Perú.

Sin embargo, como puede observar, a principios de la década de los 90, el arribo de turistas extranjeros era muy incipiente. Ello se debe a que, durante la década de los 80 , el Perú estaba sumergido en una crisis económica y social causada por la violencia terrorista que afectaba principalmente la zona sur andina, con consecuencias muy negativas para el turismo. Posteriormente, el turismo receptivo en Cuzco empieza a incrementarse y se observa que a partir de 1998 los visitantes extranjeros superan a los nacionales, esto se explica por las medidas de pacificación y la recuperación de la estabilidad económica del país. Carnaffan (2010) analiza la marca turística utilizada por PromPerú en el año 2000 "Perú país de los Incas" justamente evocando a ese pasado mágico e idealizado para utilizarlo como estrategia de diferenciación turística en el mercado global. Asimismo, a partir del año 2001, durante el gobierno de Alejandro Toledo, las políticas públicas se centraron en la promoción de la Marca País y la promoción del turismo en el mercado internacional.

Los datos también nos indican que durante 2009 y 2010, el número de turistas extranjeros disminuyó. Esta disminución se explica por varios motivos. En primer lugar, la crisis económica mundial a partir del 2008 que afectó a nuestro principal mercado emisor de turistas, como es el caso de Estados Unidos. Asimismo, el cierre por tres meses de Machu Picchu en enero de 2010, debido a los desastres que ocasionaron las lluvias y que destrozaron parte de la vía férrea que une la ciudad del Cuzco con 
la ciudadela de Machu Picchu. Este hecho afectó la imagen turística de este destino (El Comercio, 31 de enero 2010).

\section{El turismo en el contexto socioeconómico}

Si bien el número de turistas que visitan el Departamento de Cuzco ha crecido en la última década, no es la principal actividad que contribuye a generar riqueza en el departamento. Según datos del INEI (ver Gráfica 1), la minería es el primer sector que contribuye al PIB regional con 49.2\% mientras que, el sector turismo (representado por hoteles y restaurantes) ocupa el sexto lugar, con una contribución de 4.1\%.

Gráfica 1. PIB Cuzco por sectores, 2016

(Porcentaje)

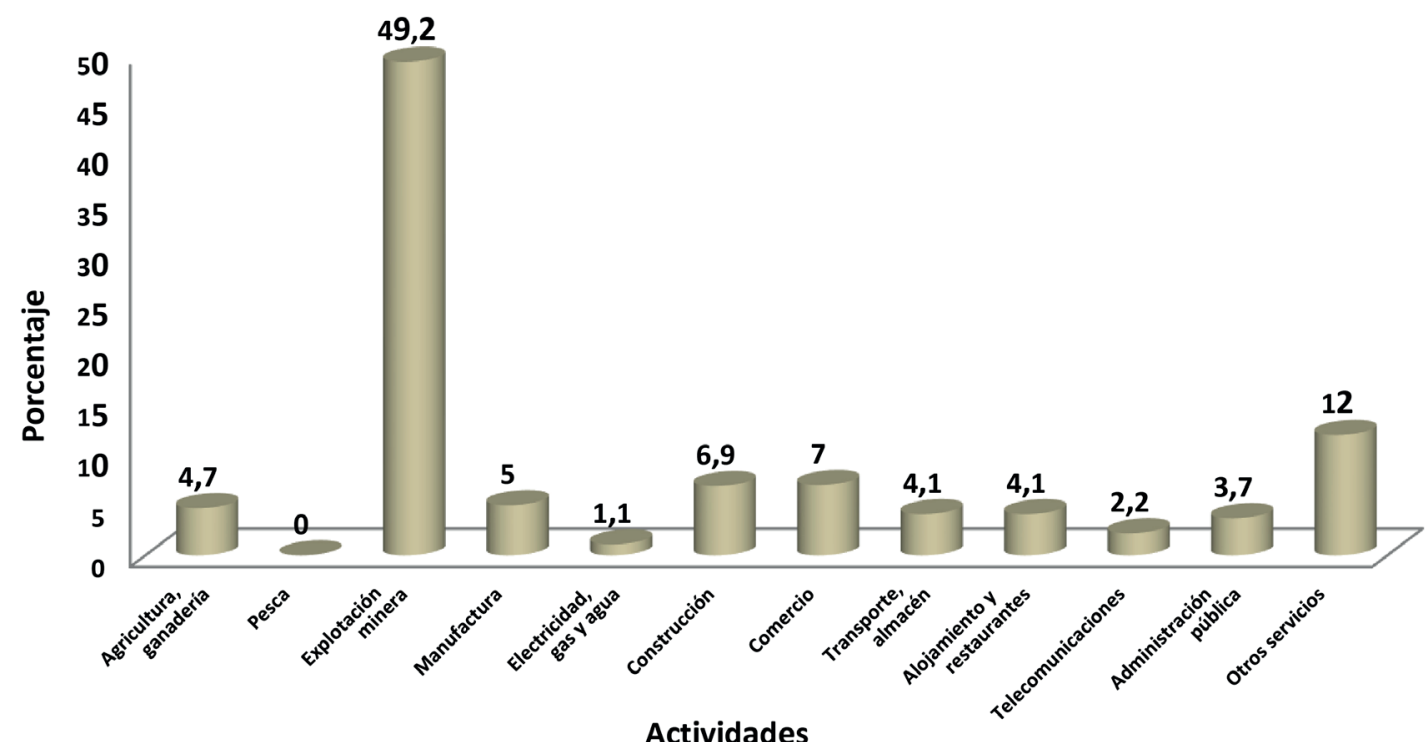

Fuente: Elaboración propia con datos de INEI (2016).

La producción minera se basa en la explotación del cobre, oro y plata. Además, Cuzco cuenta con los yacimientos de gas natural más importantes del continente americano. El Proyecto Gas de Camisea, en marcha desde el 2004, ha convertido a la región en el primer productor nacional de hidrocarburos.

Tradicionalmente la economía de Cuzco, al igual que las demás regiones andinas, se ha basado esencialmente en la agricultura; sus distintos climas favorecen su desarrollo, principalmente en el valle de Cuzco. La región posee importantes áreas de producción agrícola y agroindustrial. Asimismo, la agricultura es la fuente de subsistencia de gran parte de la población rural. En la actualidad, este sector ha reducido 
su productividad ocupando el quinto lugar, con una participación del 4.7\%. Ello se explica por la falta de tecnificación, los procesos de urbanización de Cuzco, entre otros problemas.

La región de Cuzco ha sido la región con mayor crecimiento económico en Perú. De acuerdo con los datos del INEI presentados en el cuadro 2, el crecimiento del PIB del Departamento de Cuzco se ha duplicado entre el 2007 y 2016 , pasando de 10.9 millones de soles a 21.8 millones soles en 2016. Con relación al sector turismo, si bien, la participación porcentual en el PIB regional ha ido bajando de $4.6 \%$ a $4.1 \%$ entre 2007 y 2016 , ello no significa que en términos monetarios absolutos el PIB se haya reducido; por el contrario, se ha incrementado, tal como lo ilustran los datos. Esto se explica por el crecimiento de otros sectores que están dando un mayor impulso a la economía regional.

Cuadro 2. Contribución del sector turismo al PIB del Departamento de Cuzco, 2007 y 2016

\begin{tabular}{|c|c|c|c} 
Año & $\begin{array}{c}\text { PIB Cuzco } \\
\text { (miles de soles) }\end{array}$ & $\begin{array}{c}\text { PIB turismo } \\
\text { (alojamiento y restaurantes) } \\
\text { (miles de soles) }\end{array}$ & $\begin{array}{c}\text { Participación del sec- } \\
\text { tor Turismo (\%) }\end{array}$ \\
\hline 2007 & 10913725 & 497564 & 4.6 \\
2016 & 21829280 & 888715 & 4.1 \\
\hline
\end{tabular}

Fuente: Instituto Nacional de Estadística e Informática INEI (2016).

Ahora bien, Cuzco recibe importantes ingresos por concepto de canon minero, regalías y derechos. En el periodo 2004-2007, las transferencias de canon se han incrementado notablemente, pasando de 17 millones en 2004 a 220 millones de soles en 2007, los cuales se reparten de acuerdo a la Ley del Canon (Grupo Propuesta Ciudadana, 2009, p.34), Sin embargo, hay que considerar que a partir del año 2013 se ha registrado una caída de estos ingresos por la baja de los precios internacionales de los metales, generando inconvenientes a los gobiernos municipales y las comunidades que dependen de la minería (Mendoza et al., 2014). Además, hay que tener en cuenta que la generación de empleo en el sector minero es menos intensiva. Según datos del INEl (2017, p. 269), el sector minero empleó al 0.7\% del total de la Población Económicamente Activa Ocupada en 2016.

A pesar del espectacular crecimiento económico de la región, Cuzco presenta aún graves problemas de desarrollo. Aunque en la última década se han logrado avances en la reducción de la pobreza. Según los datos del INEI que muestra el 
cuadro 3, la pobreza se redujo de $54.2 \%$ en 2007 a $25.2 \%$ en 2017 . No obstante, estos valores superan el promedio nacional. Además, la distribución de la pobreza muestra contrastes muy marcados, la capital regional y alrededores presentan menor incidencia de la pobreza que las zonas altas y montañosas. Según el Mapa de pobreza provincial y distrital 2013, elaborado por el INEI (2015), el distrito de Wanchaq, en la provincia de Cuzco, es el menos pobre de la región inferior al 0.9\%, mientras que, por ejemplo, en la zona de Paucartambo, la pobreza es mayor al $30 \%$. Es decir, la pobreza se concentra en las zonas rurales y montañosas, y el desarrollo en las ciudades o zonas urbanas.

Por otro lado, los avances han sido muy lentos en indicadores como desnutrición infantil, anemia y mortalidad infantil; en todos los casos las cifras superan el promedio nacional, lo cual evidencia un grave problema de salud pública. Con relación a la tasa de analfabetismo no hubo grandes progresos. Se observa una leve disminución de $12.1 \%$ en el 2007 a $11.5 \%$ en el 2015 ; una tasa muy superior al promedio nacional (6\%) (Ver cuadro 3).

Cuadro 3. Principales indicadores sociales del Departamento de Cuzco y Perú

\begin{tabular}{|c|c|c|}
\hline & Cuzco & Perú \\
\hline Pobreza monetaria total (\%) & & \\
\hline 2007 & 57.4 & 39.3 \\
\hline 2017 & 25.2 & 21.7 \\
\hline $\begin{array}{c}\text { Tasa de desnutrición crónica } \\
\text { niños menores de 5 años (\%) }\end{array}$ & & \\
\hline 2009 & 38.4 & 23.8 \\
\hline 2015 & 16.7 & 14.4 \\
\hline Tasa de analfabetismo & & \\
\hline de 15 a más años de edad (\%) & & 8.5 \\
\hline 2007 & 12.1 & 6 \\
\hline 2015 & 11.5 & 32.6 \\
\hline Anemia en niños menores de 5 años (\%) & & 33.3 \\
\hline 2015 & 41.7 & \\
\hline 2016 & 46.8 & 23 \\
\hline Tasa de mortalidad infantil & & \\
\hline (por cada 1000 nacidos vivos) & & \\
\hline 2014 & & \\
\hline
\end{tabular}

Fuente: INEI (2018b) can base en datos de la Encuesta Nacional de Hogares (ENAHO). 


\section{Turismo, desarrollo y lucha contra la pobreza}

Uno de los grandes problemas del desarrollo de Cuzco tiene que ver con el ineficiente nivel de ejecución del gasto público y la incapacidad de los gobiernos regional y local para ejecutar proyectos de inversión que respondan a las necesidades de desarrollo integral de la región (Sánchez Guzmán et al., 2006). Según el informe del Grupo Propuesta Ciudadana (2009), el destino de las inversiones públicas por parte del gobierno nacional entre el 2005 y 2007 ha sido principalmente en el sector transporte y comunicaciones que representa el $50 \%$ del gasto total, mientras que en el ámbito de desarrollo social se ha destinado el $18 \%$.

Otro punto esencial es la formación del capital humano. De acuerdo con Sen (2000), este acompaña el crecimiento económico y permite crear las condiciones propicias para el desarrollo sostenible. Sin ello, los agentes de desarrollo son demasiado débiles e incapaces de vencer los obstáculos que el crecimiento socioeconómico exige de todos los actores presentes en el mercado turístico. Según un estudio del Observatorio Turístico del Perú (2009) en Cuzco existe una brecha entre la oferta y la demanda de recursos humanos en el sector hotelero de la ciudad. En esta investigación el Observatorio constató la existencia de una mano de obra con muy pocos recursos educativos y con pocas posibilidades de abrirse camino hacia condiciones socioeconómicas capaces de habilitarla para nuevos emprendimientos individuales o grupales, en un mercado dominado por cadenas hoteleras foráneas (un tema analizado en Figueroa et al., 2015). En esa línea, resulta interesante la investigación de Knight, et al. (2017) sobre la comparación de los discursos de desarrollo a nivel nacional en Perú y su contraste con las visiones locales de pobreza y turismo en comunidades rurales del Valle Sagrado en Cuzco. Sus resultados demuestran que existe una confrontación constante entre el desarrollo del turismo dominado por una economía neoliberal y una mayor presión de las empresas foráneas con las visiones y necesidades de las comunidades rurales.

A todo ello hay que añadir la existencia de lo que se suele llamar informalidad, es decir la inexistencia de reglas formales que garanticen a las prácticas turísticas desarrollarse ordenada y formalmente. Según un estudio realizado por Rodríguez y Higa (2010, p. 2) acerca de la informalidad en Perú, afirma que es uno de los países con la proporción más alta de la fuerza laboral bajo alguna forma de informalidad en América Latina. Según datos del INEI (2016, p. 79) la informalidad total es de $72.3 \%$ en 2014. En cuanto a los datos de la informalidad por actividad económica en 2014, el sector restaurantes y hoteles tiene una alta incidencia de informalidad, pues casi la mitad, 40\%, de su Valor Agregado Bruto proviene del sector informal. Carlos Cana- 
les, presidente de la Cámara Nacional de Turismo (Canatur) señala que alrededor del $72 \%$ de las empresas ligadas al sector turismo son informales (Gestión, 3 de octubre 2012). Una de las razones que explica esta situación, según De Althaus (2007), es la legislación laboral que impide, por su elevado costo para las empresas, que un mayor número de empresas y trabajadores sin derechos puedan formalizarse. Por lo tanto, la informalidad no es solo un problema de gestión empresarial, sino también la consecuencia de la producción de desigualdades socioeconómicas y culturales.

\subsection{Trujillo (Departamento de La Libertad)}

El Departamento de La Libertad está situado en la costa noroccidental del Perú. Su territorio abarca las tres regiones naturales: desde las costas del Océano Pacífico, pasando por las montañas escarpadas y abruptas de la sierra andina y finalizan en las tierras altas de la selva amazónica, con una población total de 1778080 habitantes. La diversidad de su geografía y los recursos variados de la naturaleza y un clima propicio para el desarrollo de la industria agroexportadora, han atraído a la región una población donde las posibilidades de trabajo y de riqueza son más favorables, principalmente en la ciudad de Trujillo y ciudades costeras de la región.

La ciudad de Trujillo, capital del Departamento de La Libertad es actualmente uno de los destinos turísticos en pleno auge en la costa norte de Perú. La ciudad de Trujillo es una ciudad activa y dinámica, y una de las ciudades más importantes de la costa norte por sus características económicas y culturales. Desde el punto de vista económico, Trujillo es el centro económico, comercial y agrícola más importante de la región, ello se explica por el auge de las agroexportaciones y por la recuperación de las principales haciendas azucareras (De Althaus, 2007, p. 111).

Desde el punto de vista cultural, Trujillo fue territorio de importantes civilizaciones prehispánicas (Cupisnique, Moche y Chimú) del cual se conservan valiosos objetos y monumentos arqueológicos que evidencian el elevado desarrollo que alcanzaron estos pueblos. Posteriormente, con la llegada de los conquistadores españoles, Trujillo fue una importante ciudad virreinal, fundada por el conquistador Diego de Almagro en 1534. Desde ese entonces, la fisonomía de la ciudad cambió completamente. La fusión de las culturas y costumbres prehispánicas y europeas le han dado a Trujillo unas características especiales que se aprecian en la riqueza de su patrimonio histórico, la cultura y tradiciones de sus pueblos y en la variedad de su gastronomía, elementos que la hacen atractiva a la mirada turística.

En los últimos años, la región ha hecho grandes esfuerzos para diversificar su oferta turística, se ha invertido en la recuperación y puesta en valor de impor- 
tantes vestigios arqueológicos, despojados durante décadas por los huaqueros y vendedores informales de piezas arqueológicas. Gracias al esfuerzo y alianza del sector público y privado se han creado museos de vanguardia para atraer un mayor número de visitantes, y sus atractivos se han articulado desde 2008 en la ruta turística denominada Ruta Moche que incluye los Departamentos de La Libertad y Lambayeque para promocionar de manera conjunta los atractivos turísticos de la costa norte de Perú.

Los íconos arqueológicos más representativos de la región son: la ciudadela de Chan Chan, declarada Patrimonio de la Humanidad por la Unesco en 1986, al ser la ciudad de adobe más grande de América prehispánica; las Huacas del Sol y La Luna; el descubrimiento (en 2006) de la Señora de Cao, en el Complejo Arqueológico El Brujo, que según los estudios realizados se trataría de la primera mujer gobernante de la cultura Moche. Estos atractivos se complementan con playas como el balneario y pueblo de Huanchaco o tradiciones culturales tales como su famoso festival de La marinera.

\section{Crecimiento del turismo}

La demanda turística hacia la ciudad de Trujillo se ha duplicado en el periodo 19922015. Sin embargo, Trujillo como destino turístico atrae más al turista nacional que al extranjero. Según datos del Observatorio Turístico del Perú, que se presentan en el cuadro 4, el departamento recibió en 2015 un total de 623 mil 382 turistas, de los cuales 567 mil 476 son turistas nacionales y 55 mil 536 turistas extranjeros. Incluso se observa que la región recibe más turistas nacionales que Cuzco y los motivos son fácilmente comprensibles: la ciudad de Trujillo es más accesible en términos económicos para el turista nacional que Cuzco. Y como Trujillo está ubicada en la costa, el acceso es más fácil. 
Cuadro 4. Departamento de La Libertad: Evolución del número de turistas 1992-2015

\begin{tabular}{|c|c|c|c|}
\hline Año & $\begin{array}{c}\text { Turismo Receptor } \\
\text { Histórico }\end{array}$ & $\begin{array}{c}\text { Turismo Interno } \\
\text { Histórico }\end{array}$ & Total \\
\hline 1992 & 4069 & 306564 & 310633 \\
\hline 1993 & 8318 & 316658 & 324976 \\
\hline 1994 & 25749 & 628717 & 654466 \\
\hline 1995 & 52598 & 767924 & 820522 \\
\hline 1996 & 61346 & 857035 & 918381 \\
\hline 1997 & 51197 & 793809 & 845006 \\
\hline 1998 & 24935 & 587540 & 612475 \\
\hline 1999 & 31091 & 556639 & 587730 \\
\hline 2000 & 27515 & 460190 & 487705 \\
\hline 2001 & 32955 & 452150 & 485105 \\
\hline 2002 & 28379 & 445838 & 474217 \\
\hline 2003 & 39612 & 563407 & 603019 \\
\hline 2004 & 40939 & 563777 & 604716 \\
\hline 2005 & 42266 & 564147 & 606413 \\
\hline 2006 & 43593 & 564517 & 608110 \\
\hline 2007 & 44920 & 564887 & 609807 \\
\hline 2008 & 46247 & 565257 & 611504 \\
\hline 2009 & 44735 & 565627 & 610362 \\
\hline 2010 & 48901 & 565997 & 614898 \\
\hline 2011 & 50228 & 566367 & 616595 \\
\hline 2012 & 51555 & 566737 & 618292 \\
\hline 2013 & 52882 & 567106 & 619988 \\
\hline 2014 & 54209 & 567476 & 621685 \\
\hline 2015 & 55536 & 567846 & 623382 \\
\hline
\end{tabular}

Fuente: Elaboración propia con datos del Observatorio Turístico del Perú.

Llama la atención que, a mediados de la década de 1990, el crecimiento del turismo fue espectacular, llegando a recibir en total algo más de 918 mil visitantes en 1996. Los visitantes extranjeros aumentaron considerablemente ese mismo año. Sin embargo, los años siguientes descendió notablemente, no llegando a superar en la actualidad la cifra mencionada. Intentemos dar una explicación de estos insólitos hechos.

La primera idea es que, entre 1996 y 1998, el fenómeno del Niño afectó todo Perú y produjo importantes catástrofes en los Andes del Sur, siendo muy afectada 
la región del Cuzco/Machu Picchu (Corporación Andina de Fomento, 2010). Ese hecho devastador tuvo efectos muy dañinos en el Sur, pero se volvió una bonanza en vastas regiones de la Costa en términos turísticos. Es decir, el Sur dejó de recibir sus visitantes y la Costa aprovechó esa circunstancia. Es, por cierto, una explicación catastrófica; no obstante, es muy realista para el país. Probablemente es el mismo fenómeno que da razón de la desaparición de culturas tan ricas y prósperas como la de Los Moches.

Con el objetivo de convertir la Ruta Moche en el segundo destino turístico internacional en Perú, el Mincetur destinó 11 millones de soles en 2008 para la ejecución de obras e infraestructura turística en el norte de Perú. Asimismo, el gobierno a través de la Comisión de Promoción del Perú para la Exportación y el Turismo (PromPerú) desarrolló un plan de marketing para incrementar el turismo internacional, con un presupuesto de 4 millones de soles para 2011 (Andina, 10 de agosto 2010). Lo cierto es que, pese a los esfuerzos hasta el momento, la Ruta Moche no ha logrado el impacto esperado. Los datos demuestran que el número de visitantes extranjeros al Departamento de La Libertad no registra un aumento notable (La República, 14 de julio 2014; Tnews, 2016).

\section{Las practicas turísticas en el espacio económico}

Si bien el turismo en la región es un sector dinámico gracias a la puesta en valor de su patrimonio, la participación del Turismo en el PIB regional, en términos porcentuales, no tiene peso muy importante. Los datos disponibles del INEI que se presentan en la Gráfica 2, demuestran que el sector turismo (representado por hoteles y restaurantes) ocupa el noveno lugar en contribución al PIB regional, con un aporte de $2.5 \%$ en 2016. 
Gráfica 2. PIB del Departamento de La Libertad por sectores, 2016

(Porcentaje)

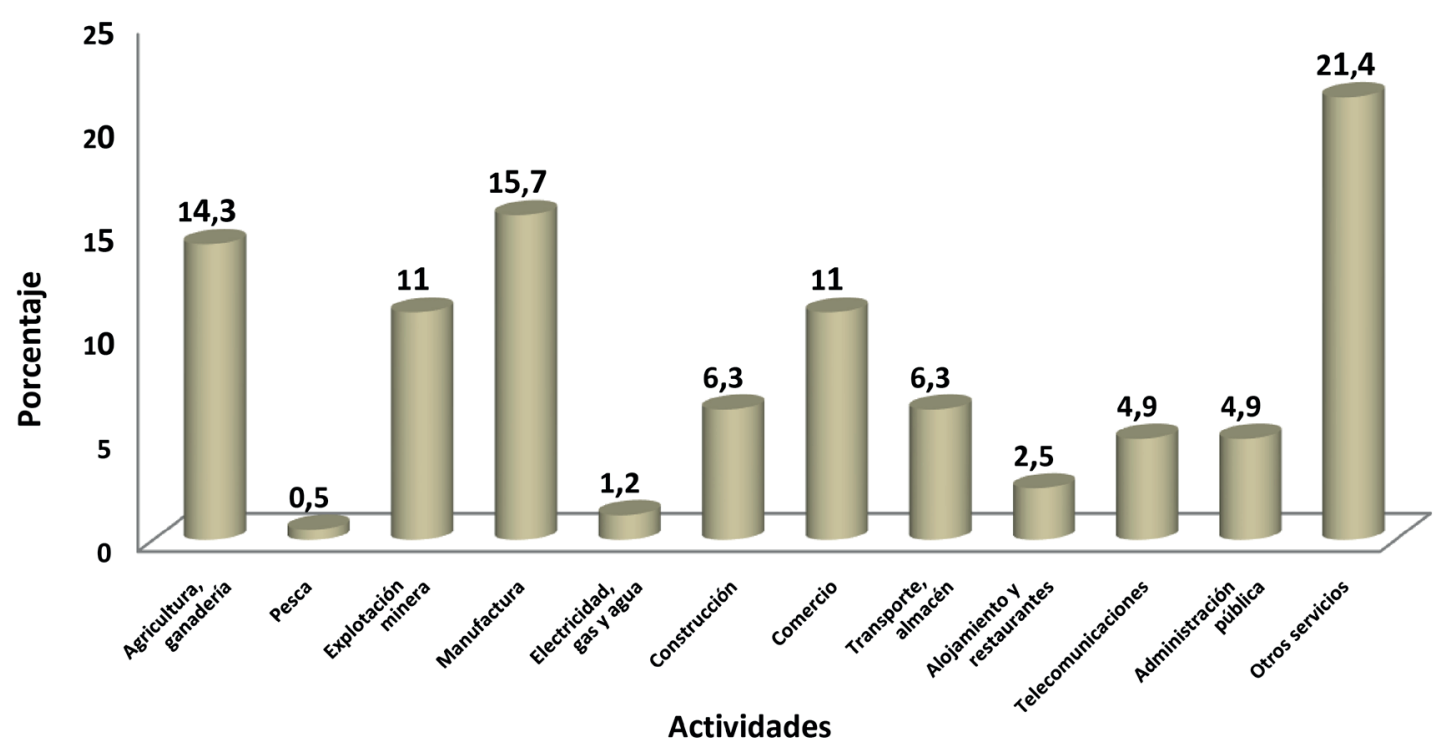

Fuente: Elaboración propia con datos estadísticos del INEI (2016).

Ello explica por qué otros sectores han crecido y adquirido mayor relevancia en la economía regional. En primer lugar, la manufactura que aporta $15.7 \%$ y en segundo lugar la agricultura con una participación de $14.3 \%$. La minería es la cuarta actividad más importante con un aporte de $11 \%$ al PIB regional y produce principalmente oro, entre otros minerales. Esta actividad permite a la región recibir ingresos por concepto de transferencia de canon minero que en 2016 registró 253 millones de soles (El Comercio, 1 de agosto 2017).

\section{Pobreza y prácticas turísticas}

La región presenta un nivel de desarrollo más elevado que el resto de las regiones peruanas y se encuentra en un nivel intermedio en cuanto a niveles de pobreza y desarrollo humano. Según datos del INEI 82017), que se aprecian en el cuadro 5, la pobreza monetaria total se ha reducido de $37.3 \%$ en el 2007 a $23.5 \%$ en el 2017 , aunque aún supera el promedio nacional.

Los datos económicos dan muestra del crecimiento favorable y dinámico especialmente en la costa, precisamente en la ciudad de Trujillo mientras que las poblaciones de las zonas periféricas y en las provincias de la sierra tienen condiciones de vida más precarias. Por ejemplo, según el Mapa de Pobreza Provincial y Distrital elaborado 
por el INEI en el 2009, la provincia con menor pobreza es Trujillo con $21.5 \%$ de pobreza total. Dentro de la provincia de Trujillo, el distrito de Moche (donde se ubica el complejo arqueológico Huacas de Moche), la pobreza alcanza al 33.7\% de la población. Mientras en las provincias más alejadas como Julcán la pobreza total alcanza el 77\% de la población. Esa diferencia de desarrollo, como señala Cotler et al. (2009) puede deberse a una marcada desintegración del territorio por la falta de infraestructura vial que conecte la costa con el interior de la región.

Con relación a indicadores sociales de salud y educación, se observa que la región ha hecho grandes progresos en comparación a Cuzco. Por ejemplo, la tasa de analfabetismo ha disminuido de $8.3 \%$ en 2009 a $6 \%$ en 2015, igual al promedio nacional. Aunque todavía queda mucho por mejorar, en cuanto a niveles de desnutrición y mortalidad infantil que muestran niveles que superan el promedio nacional (ver cuadro 5).

Cuadro 5: Principales indicadores sociales del Departamento de La Libertad y Perú

\section{La Libertad}

\section{Pobreza monetaria total (\%)}

2007

2017

Tasa de desnutrición crónica niños menores de 5 años (\%)

2009

2015

Tasa de analfabetismo de 15 a más años de edad (\%)

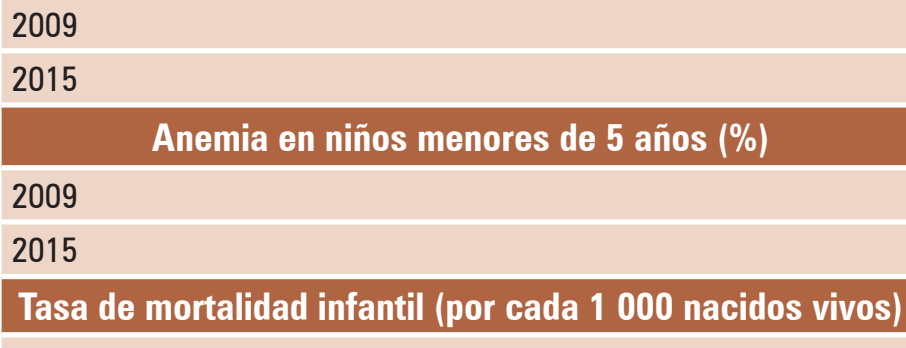
2014

\begin{tabular}{|l|l|}
\hline 37.3 & 39.3 \\
\hline 23.5 & 21.7 \\
\hline
\end{tabular}

\begin{tabular}{l|l}
27.2 & 23.8 \\
15.9 & 14.4
\end{tabular}

\begin{tabular}{|l|l|}
\hline 8.3 & 7.6 \\
\hline 6.0 & 6.0 \\
\hline & \\
\hline 36.9 & 37.2 \\
\hline 24.7 & 32.6 \\
\hline
\end{tabular}

24

23

Fuente: Elaboración propia con datos del INEI (2017).

En ese sentido la puesta en valor del patrimonio arqueológico y cultural de la región ha significado un importante beneficio y una oportunidad para revalorar la identidad e integrar a la población local en la gestión de las actividades turísticas. Por ejemplo, 
Morales Gamarra (2010) director del proyecto arqueológico Huaca de la Luna, explica que este proyecto cuenta con un programa importantísimo para la región, porque abarca cuatro áreas específicas: arqueología, conservación y acondicionamiento, uso público y desarrollo comunitario. Estos cuatro aspectos conforman una nueva estrategia de gestión y manejo pensando en beneficiar a la comunidad local. Y, de hecho, según el autor, este proyecto es un polo de desarrollo económico y social al haber generado puestos de trabajo directo e indirecto que favorecen a la población local. Morales Gamarra también explica que el $90 \%$ de los ingresos generados por concepto de visitas son reinvertidos en las actividades del proyecto y en obras públicas a favor de la comunidad.

Por otra parte, Trivelli y Hernández (2009) critican la reducida inversión por parte de las autoridades locales en los proyectos arqueológicos de la región. También señalan la falta de involucramiento por parte del Ministerio de Cultura limitándose principalmente a aspectos normativos, dejando mayor libertad a las fundaciones privadas en la gestión del patrimonio. Por lo tanto, en el caso de las Huacas de Moche y Magdalena de Cao el mayor grado de involucramiento lo integran las fundaciones nacionales y extranjeras, y en menor medida el Gobierno central y regional.

\section{Perspectivas comparativas generales}

Ambas regiones analizadas tienen rasgos comunes, por ejemplo: grandes riquezas en recursos patrimoniales, arqueológicos y naturales, con una base demográfica amplia. En los últimos 15 años han experimentado un crecimiento económico espectacular gracias a la explotación de recursos minerales y grandes proyectos agroindustriales. En ambos casos no son las prácticas turísticas que sirven de motor principal a la producción de riqueza regional, ni siquiera en el eje Cuzco/Machu Picchu. Las inversiones mineras y la agricultura prevalecen si las comparamos con el sector turismo. Como resultado de ello, los dos departamentos analizados, sobre todo Cuzco, reciben sumas importantes de dinero a través del llamado "canon minero".

Ahora bien, las actividades mineras están sometidas a un régimen fiscal que las obliga a otorgar al Estado y a las regiones donde se encuentran ciertos réditos - canon minero - mientras las actividades turísticas recogen sus beneficios por impuesto común y corriente, de tal manera que la riqueza generada por las prácticas turísticas obedece simple y llanamente a las reglas del mercado. El Ministerio de Cultura administra una parte importante de los ingresos por concepto de boletos a los sitios arqueológicos e impuestos locales, que son reinvertidos en su mantenimiento. 
Ambas regiones tienen problemas persistentes de pobreza y desarrollo social. La pobreza se concentra en el ámbito rural donde la población experimenta profundas privaciones. En ambos casos, como lo prueban las estadísticas analizadas, son las decisiones políticas las que van a determinar el grado de inversión en el desarrollo social de estas regiones.

El caso más preocupante corresponde a Cuzco, principal destino turístico internacional en Perú. Las enormes deficiencias en salud y educación no permiten a la población participar cabalmente y sacar provecho de la riqueza producida por el turismo, relegando a parte de la población hacia los espacios de la informalidad y de la supervivencia, engrosando los grupos sociales considerados pobres o por debajo de los niveles de pobreza. Por lo tanto, la relación entre informalidad y pobreza amerita una investigación más profunda.

En el Departamento de La Libertad y precisamente en la ciudad de Trujillo las condiciones socioeconómicas en general son más aceptables. Tiene menores niveles de pobreza socioeconómica. Su nivel de desarrollo educativo es más alto que los encontrados en Cuzco. No obstante, existe una importante bolsa de población socioeconómicamente pobre, generalmente ubicada en las zonas periféricas de la ciudad y en los alrededores de los centros arqueológicos más importantes. En ese sentido, el crecimiento del turismo y la puesta en valor de sus monumentos arqueológicos pueden significar un aporte importante para reducir la pobreza de las comunidades locales en los próximos años.

\section{Conclusiones}

Las lecciones obtenidas con base en el análisis de ambos casos en Perú demuestran la compleja relación entre el turismo y la pobreza; es decir, el incremento del turismo no va a resolver automáticamente los preocupantes problemas sociales, tal como lo señala el discurso generalizado de los organismos internacionales. Por lo tanto, las prácticas turísticas aún no pueden ser consideradas como el gran motor del desarrollo socioeconómico de las respectivas regiones.

En ese sentido, las regiones que no permiten o dificultan el acceso a la educación y a los bienes y servicios elementales son víctimas de su propio abandono. No se trata, pues, solamente de crear las condiciones para que las prácticas turísticas puedan llevarse a cabo en una región, sino más bien de proporcionar a los individuos o grupos los instrumentos de su propio desarrollo y capacitarlos para que el desarrollo 
sea parte integrante de su porvenir como individuos o grupos activos y dinámicos en la región.

Perú tiene un enorme desafío en lograr que el crecimiento del turismo se integre a un proyecto de desarrollo territorial, que contribuya a la lucha contra la pobreza, desarrollando espacios humanos de forma sostenible principalmente en las zonas rurales laissées pour compte.

\section{Referencias}

Alkire, S. y Foster, J. (2007). Counting and Multidimensional Poverty Measurement, OPHI Working Paper 7(32), 1-44. Recuperado de https://bit.ly/3eAb8ir

Andina (10 de agosto de 2010). Impulsarán Ruta Moche como segundo destino turístico del Perú. Andina-Agencia Peruana de Noticias. Recuperado de https:// bit.ly/2xwGHcE

Carnaffan, S. J. (2010). Peru: Land of the Incas? Development and Culture in Responsible, Homestay Tourism in Peru (tesis de doctorado). Newcastle University, Inglaterra, Reino Unido.

Christin, R. (2010). Manuel de l'antiturisme. Montreal, Canadá: Éditions Écososiété Corporación Andina de Fomento (2010). El Fenómeno del Niño 1997 - 1998 memoria, retos y soluciones. Vol. V: Perú. CAF. Recuperado de https://bit.ly/2Vmy0L4

Cotler, J. Barrenechea, R. Glave, M. Grompone, R. y Remy, M. I. (2009). Poder y Cambio en las Regiones. Lima, Perú: IEP, PNUD (Cuadernos PNUD. Serie Desarrollo Humano $\left.\mathrm{N}^{\circ} 15\right)$.

De Althaus, J. (2007). La revolución capitalista en el Perú. Lima, Perú: Fondo de Cultura Económica.

Dehoorne, O. (2013). Tourisme et lutte contre la pauvreté: opportunités et défis. Revue Études Caribéennes, 24-25. Recuperado de https://bit.ly/3crZkgv

De Kadt, E. (1991). Turismo: ¿pasaporte al desarrollo?: Perspectivas sobre los efectos sociales y culturales del turismo en los países en vías de desarrollo. Madrid, España: Endymion.

El Comercio. (31 de enero 2010). Cusco en crisis: Machu Picchu requiere una decisión política para evitar desastre. Recuperado de https://bit.ly/2RJvfkH

El Comercio. (1 de agosto de 2017). Regiones recibieron S/ 1.862, 7 millones por canon minero. Recuperado de https://bit.ly/3exS58u

Figueroa, J., Arellano, A. y Tello, S. (2015). Développement touristique ou reproduction sociale de la pauvreté: Les leçons de Cuzco, Pérou, Téoros, 33(2), 1-47. Recuperado de http://journals.openedition.org/teoros/2679 
Gascón, J. (2012). Introducción: Apuntes para un análisis crítico del turismo. En J. Buades, E. Cañada y J. Gascón, El turismo en el inicio del milenio: una lectura crítica a tres voces (pp.11-21). Madrid: Foro Turismo Responsable.

Gascón, J. y Cañada, E. (2005). Viajar a todo tren: turismo, desarrollo y sostenibilidad. Barcelona, España: Icaria Editorial.

Gascón, J., Morales, S. y Tresserras, J. (Eds.) (2013). Cooperación en turismo. Nuevos desafíos, nuevos debates. Barcelona, España: Foro de Turismo Responsable.

Gestión. (3 de octubre de 2012). El 72\% de las empresas ligadas al turismo es informal. Recuperado de https://bit.ly/2VDJJ6S

González Velarde, F. (2017). Estado y modelo de desarrollo turístico en la costa Norte del Perú: El caso de Máncora, Piura, Pasos, 15(4), 867-88. doi: https://doi. org/10.25145/j.pasos.2017.15.059

Goodwin, H. (2013). Turismo y reducción de la pobreza. En J. Gascón, S. Morales y J. Tresserras (Eds.), Cooperación en turismo. Nuevos desafíos, nuevos debates (pp. 387-405). Barcelona, España: Foro de Turismo Responsable.

Grupo Propuesta Ciudadana (2009). Gasto público y canon en el Perú. Análisis y recomendaciones para el mejor aprovechamiento de las rentas del Gas de Camisea, Lima, Perú: Grupo de Propuesta Ciudadana, CBC. Recuperado de https://bit.ly/34OLcvm

Hawkins, D. E. y Mann, S. (2007). The World Bank's role in tourism development. Annals of Tourism Research, 34(2), 348-363.

Herrera, J. (2002). Introducción. Pobreza y desigualdad en el área andina. Elementos para un nuevo paradigma. Bulletin de l'Institut Français d'Études Andines, $31(3), 413-428$.

Huaita Alfaro, A. M. (2012). Ecoturismo y desarrollo humano: el caso de una asociación privada con la comunidad de infierno, Turismo y Patrimonio, 7, 23-31.

Hummel, J. y Van der Duim, R. (2012). Tourism and development at work: 15 years of tourism and poverty reduction within the SNV Netherlands development organisation. Journal of Sustainable Tourism, 20(3), 319-338.

Instituto Nacional de Estadística e Informática (INEI). (2015). Mapa de pobreza distrital y provincial 2013, Lima, Perú: INEl. Recuperado de https://bit.ly/2VnGaTv

Instituto Nacional de Estadística e Informática (INEI). (2016). Producción y empleo informal en el Perú. Cuenta satélite de la economía informal 2007-2015. Lima, Perú: INEI. Recuperado de https://bit.ly/3el76F4

Instituto Nacional de Estadística e Informática (INEI). (2017). La Libertad compendio estadístico 2017. Lima, Perú: INEl. Recuperado de https://bit.ly/2yvy5mn 
Instituto Nacional de Estadística e Informática (INEI). (2018a). Perú: Crecimiento y distribución de la población, 2017. Primeros resultados. Junio 2018. Lima, Perú: INEI. Recuperado de https://bit.ly/2RMIYai

Instituto Nacional de Estadística e Informática (INEI). (2018b). Evolución de la pobreza monetaria 2007-2017. Informe Técnico. INEl. Recuperado de https://bit. ly/2RNevZV

Jurdao Arrones, F. (1992). Los mitos del turismo. Madrid, España: Ediciones Endymion.

Knight, D.W., Cottrell, S.P., Pickering, K., Bohren, L y Bright, A. (2017) Tourism-based development in Cusco, Peru: comparing national discourses with local realities, Journal of Sustainable Tourism, 25(3), 344-361, DOI: 10.1080/09669582.2016.1213269

La República. (14 de julio 2014). Ruta Moche aún no logra ser el segundo destino turístico del país. Recuperado de https://bit.ly/34Npnfq

Lima, S., García, C. y Eusébio, C. (2012). El turismo como una estrategia para el mundo en desarrollo: el Programa UNWTO. Volunteers. Pasos, Revista de Turismo y Patrimonio Cultural, 10(3), 303-314.

Lima, S. (2014). International Cooperation for Development in Tourism Destinations (tesis de doctorado). Universidad de Aveiro, Aveiro, Portugal.

Mendoza N. A., Passuni P. S. y De Echave, J. (2014). La minería en el Sur Andino: el caso de Cusco. Lima, Perú: CooperAction- Acción solidaria para el desarrollo.

Ministerio de Comercio Exterior y Turismo (Mincetur). (2016). Plan Estratégico Nacional de Turismo 2025 (Pentur). Lima, Perú: Mincetur. Recuperado de https:// bit.ly/2VkUEDC

Ministerio de Comercio Exterior y Turismo (Mincetur). (s.f). Estadísticas en línea. Recuperado de http://www.mincetur.gob.pe

Morales Gamarra, R. (2010). Entre el Sol y La Luna: Huacas de Moche, arqueología, conservación y desarrollo. Gaceta Cultural del Perú, (41), 12-15.

Observatorio Turístico del Perú. (2009). Badatur estadísticas. Recuperado de http:// www.observatorioturisticodelperu.com

Organización Mundial del Turismo (OMT). (2003). Turismo y atenuación de la pobreza. Madrid, España: OMT.

Organización Mundial del Turismo (OMT). (2005). Turismo, microfinanzas y reducción de la pobreza. Madrid, España: OMT -PlaNet Finance.

Organización Mundial del Turismo (OMT). (2010). Manual on Tourism and Poverty Alleviation - Practical Steps for Destinations. Madrid, España: OMT. 
Rodríguez, J. e Higa, M. (2010). Informalidad, empleo y productividad en el Perú. (Documento de trabajo $\mathrm{n}^{\circ}$ 282). Lima, Perú: Pontificia Universidad Católica del Perú. Recuperado de https://bit.ly/2RO2CTu

Sánchez Guzmán, E., Muennekhoff, B. y Ricard Lanata, X. (2006). Propuesta para el gobierno regional 2007-2010. Región Cusco. Lima, Perú: CIES / CBC.

Scheyvens, R. (2007). Exploring the Tourism-Poverty Nexus. Current Issues in Tourism, 10(2-3), 231-254.

Scheyvens, R. y Russell, M. (2012). Tourism and poverty alleviation in Fiji: comparing the impacts of small- and large-scale tourism enterprises. Journal of Sustainable Tourism, 20(3), 417-436.

Sen, A. (2000). Desarrollo y libertad. Barcelona, España: Editorial Planeta.

Tnews (22 de agosto 2016). Cifra de turistas en la Ruta Moche no despega. Recuperado de https://bit.ly/3afxzpW

Trivelli, C. y Hernández Asencio, R. (2009). Apostando por el desarrollo territorial rural con identidad cultural: La puesta en valor del patrimonio prehispánico de la costa norte de Perú. En Ranaboldo, C. y Schejtmann, A. (Eds.), El valor del patrimonio cultural. Territorios rurales, experiencias y proyecciones latinoamericanas (pp. 201-307). Lima, Perú: IEP, Centro Latinoamericano para el Desarrollo Rural.

United Nations Development Programme (UNDP). (2011). Tourism and Poverty Reduction Strategies in the Integrated Framework for Least Developed Countries. Discussion paper. Recuperado de https://bit.ly/2xwFpyk

Urry, J. (2004). La mirada del turista. Lima, Perú: Universidad de San Martín de Porres. Valcárcel, L. E. (2009). Machu Picchu. El más famoso monumento arqueológico del Perú. Lima, Perú: Fondo de Cultura Económica del Perú. 